NASA Technical Memorandum 106356

ICOMP-93-34

$1 N-34$

AIAA-93-2380

Development of an Explicit Multiblock/Multigrid

Flow Solver for Viscous Flows in Complex Geometries

185373

179

$=$

E. Steinthorsson

Institute for Computational Mechanics in Propulsion

Lewis Research Center

Cleveland, Ohio

and

M.S. Liou and L.A. Povinelli

National Aeronautics and Space Administration

Lewis Research Center

Cleveland, Ohio

Prepared for the

29th Joint Propulsion Conference and Exhibit

cosponsored by the AIAA, SAE, ASME, and ASEE

- . . . Monterey, California, June 28-30, 1993
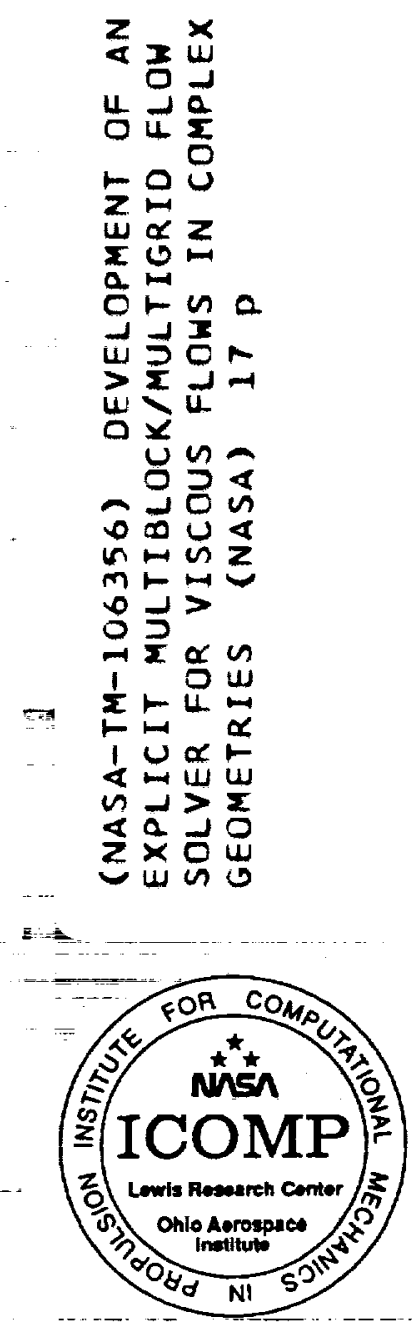



\title{
DEVELOPMENT OF AN EXPLICIT MULTIBLOCK/MULTIGRID FLOW SOLVER FOR VISCOUS FLOWS IN COMPLEX GEOMETRIES
}

\author{
E. Steinthorsson" \\ Institute for Computational Mechanics in Propulsion, ${ }^{* *}$ \\ NASA Lewis Research Center, Cleveland, Ohio \\ and \\ M.-S. Liou $\dagger \quad$ and $\quad$ L.A. Povinelli $\ddagger$ \\ Internal Fluid Mechanics Division, \\ NASA Lewis Research Center, Cleveland, Ohio
}

\begin{abstract}
A new computer program is being developed for doing accurate simulations of compressible viscous flows in complex geometries. The code employes the full compressible Navier-Stokes equations. The eddy viscosity model of Baldwin and Lomax is used to model the effects of turbulence on the flow. A cell centered finite volume discretization is used for all terms in the governing equations. The Advection Upwind Splitting Method (AUSM) is used to compute the inviscid fluxes, while central differencing is used for the diffusive fluxes. A four-stage Runge-Kutta time integration scheme is used to march solutions to steady state, while convergence is enhanced by a multigrid scheme, local timestepping and implicit residual smoothing. To enable simulations of flows in complex geometries, the code uses composite structured grid systems where all grid lines are continuous at block boundaries (multiblock grids). Example results are shown a flow in a linear cascade, a flow around a circular pin extending between the main walls in a high aspect-ratio channel, and a flow of air in a radial turbine coolant passage.
\end{abstract}

\section{Introduction}

In general, flow solvers that rely on single-block, boundary conforming, structured grid systems can only simulate flows in relatively simple geometries. For simulation of flows in geometries of arbitrary complexity, alternative grid structures must be used. The grid structures that have been used in past years for com-

\footnotetext{
*Senior Research Associate, Member AIAA

** located at the Ohio Aerospace Institute adjacent to the NASA Lewis Research Center

tsenior Scientist, Member AIAA.

$\ddagger$ Deputy Chief, Fellow AIAA
}

plex geometries can be broadly classified as (a) unstructured and hybrid structured/unstructured grids (b) completely discontinuous composite grids (Chimera grids ) and partially discontinuous composite structured grids, and (c) multiblock grids-i.e., composite grids with continuous grid lines at block boundaries (see Ref. 1 on classification of grid systems). It is worthwhile to review briefly the advantages and disadvantages of each grid structure.

Grid systems in the first category, i.e., unstructured or hybrid structured/unstructured grids are considered by many to be the type of grids that will in the long run become the grid structure of choice for complex geometries. The reason behind this belief is that algorithms exist that make the generation of unstructured grids almost completely automatic, requiring only minimal human intervention. Furthermore, unstructured grids can relatively easily be made solution adaptive and thus promise high resolution of small scale features in the computed solutions as well as the geometries. However, while computations of inviscid flows on unstructured grids have met considerable success, computation of high Reynolds number viscous flows has to date only been moderately successful. ${ }^{2-4}$

Compared to using unstructured grid, the advantage of using structured grids is that current algorithms for the Navier-Stokes equations yield better solutions for high Reynolds number viscous flows on structured, body-fitted grid systems than on unstructured grids. In addition, structured flow solvers are typically more effcient than unstructured flow solvers due to the inherent regularity in the data structures that can be used. However, the disadvantage of relying on structured grids is that it has traditionally been a difficult and labor intensive process to generate high quality structured grids for even moderately complex geometries.

To reduce the difficulty in generating structured grids for complex geometries some researchers have opted for using completely discontinuous composite grids (Chimera grids) where the individual grid blocks are al- 
lowed to overlap in an arbitrary manner. However, using Chimera grids simply shifts the difficulty in dealing with the complex geometries from the grid generation to the flow solver. To deal with the irregularly overlapping grids in the flow solver, complicated interpolation schemes are needed to accurately transfer data between blocks. The complexity of the interpolation schemes increases rapidly with the order of accuracy that is used. At the same time, if the interpolations are not done with sufficient accuracy, spurious phenomena such as reflection of waves can be observed in the computed solutions at interfaces between blocks.

The type of composite structured grid systems that lead to least complications in flow solvers are the multiblock grids. Since no interpolations are needed at the block interfaces in multiblock grids, exchange of data between blocks is relatively straightforward and no errors are generated. On the other hand, high quality multiblock grids have traditionally been the most difficult and labor intensive to generate. The source of the difficulty has been a total lack of automation in generating suitable block structures (topologies) for complex geometries. Due to this difficulty, reliance on multiblock grid systems has often been rejected in the past. Lately, however, several methods and software have emerged that aid in the decomposition of a complex geometry into blocks or zones in such a manner that high quality grid systems with good orthogonality and smoothness can be generated for the entire geometry (see, e.g., Fig. 1)..$^{5-8}$ The emergence of these methods and software has made multiblock grids a viable alternative for geometries of arbitrary complexity.

It is the objective of this ongoing research effort to develop a new computer code that is capable of simulating flows in complex geometries with high accuracy and efficiency. To achieve this goal, the decision was made to employ multiblock grid systems in the code. The basis for this decision is that the new grid generation software promises to deliver high quality grid systems with less human involvement than ever before, and that high Reynolds number viscous flows are best computed on body-fitted structured grid systems.

The new multiblock code developed in this study is derived from an existing flow solver, TRAF3D.9,10 TRAF $3 D$ is a highly efficient single-block, multigrid flow solver that was written specifically for flows in turbomachinery cascades. Unlike TRAF3D, which uses central differencing with artificial dissipation for the convection terms of the governing equations, the new code uses a recently developed upwind scheme, the Advection Upwind Splitting Method (AUSM). ${ }^{11}$ The AUSM scheme is a relatively simple but highly effective upwind scheme. It is effective in shock capturing while yielding low artificial dissipation at low Mach numbers, and it resolves stationary discontinuities exactly.

The remainder of this paper describes the essential features of the new computer code which will henceforth be referred to as TRAF3D.MB. The paper is organized as follows: Immediately following this introduction, the governing equations used in TRAF3D.MB and their discretization will be described. Afterwards, the time stepping scheme and convergence acceleration techniques that are employed will be briefly discussed. Then, the multiblock strategy employed in TRAF3D.MB will be described in some detail. Finally, results from sample calculations will be presented. The paper ends with some concluding remarks.

\section{Governing Equations and Discretization}

The governing equations employed in the TRAF$3 D . M B$ code are the compressible Navier-Stokes equations; i.e., the continuity equation, the momentum equations, and total energy equation (see, e.g., Ref. 12). The fluid is assumed to be a thermally and calorically perfect gas. The effect of turbulence on the flow are modeled by using the Baldwin-Lomax turbulence model. ${ }^{9,13}$.

In TRAF3D.MB, all terms of the governing equations are discretized by using cell-centered finite volume formulation. A recently developed upwind scheme, the advection upwind splitting method (AUSM) ${ }^{11}$ is used for the convection terms of the governing equations. The diffusion terms are discretized in a conservative fashion using second order accurate, centered finite difference formulas for the diffusive fluxes. Boundary conditions are implemented using ghost cells (also called phantom cells or auxiliary cells). At subsonic inflow boundaries, the flow direction, total pressure and total enthalpy are specified, whereas the outgoing Riemann invariant is extrapolated from the interior. At subsonic outflow boundaries, the static back pressure is specified but density and velocity are extrapolated. Either zeroth-order or first-order extrapolation can be used at the outflow boundaries. All boundary conditions in TRAF3D.MB are specified conveniently and logically at run time through NAMELIST input.

The complete details of the governing equations and discretizations used in TRAF3D.MB will not be given in this paper. Here, only the formulation of the AUSM scheme will be given as it represents a modification from the discretization that was used in its predecessor, TRAF3D. Readers interested in further details are referred to Ref. 9 which describes the formulation used in the original code. 
The formulation of the AUSM scheme for a general stationary grid system is as follows: Consider a cell face with unit normal $\vec{n}=\left(n_{x}, n_{y}, n_{z}\right)$. The state of the fluid on the "left" and "right" sides of the cell face is given by $U_{L}$ and $U_{R}$, respectively, where

$$
U=\left(\begin{array}{c}
\rho \\
\rho u \\
\rho v \\
\rho w \\
\rho(e+p / \rho)
\end{array}\right)
$$

and, $\rho, u, v, w, e$, and $p$ denote density, $x-, y-$, and $z$-components of velocity, total energy per unit mass, and pressure. Corresponding to the "left" and "right" states, define local "directional" Mach numbers as

$$
M_{L}=\frac{\vec{u}_{L} \cdot \vec{n}}{a_{L}} \quad M_{R}=\frac{\vec{u}_{R} \cdot \vec{n}}{a_{R}}
$$

where $\vec{u}=(u, v, w)$ and $a=\sqrt{\gamma p / \rho}$. The inviscid flux through a unit area of the cell face is then computed as a function of $U_{L}, U_{R}$, and $\vec{n}$ as

$$
F\left(U_{L}, U_{R}\right)=F^{+}+F^{-}
$$

where

$$
\begin{gathered}
F^{+}=M_{c}^{+} a_{L} U_{L}+M_{p}^{+} p_{L}\left(\begin{array}{c}
0 \\
n_{x} \\
n_{y} \\
n_{z} \\
0
\end{array}\right) \\
F^{-}=M_{c}^{-} a_{R} U_{R}+M_{p}^{-} p_{R}\left(\begin{array}{c}
0 \\
n_{x} \\
n_{y} \\
n_{z} \\
0
\end{array}\right) \\
M_{c}^{+}=\frac{1}{2}\left(m^{+}+m^{-}+\left|m^{+}+m^{-}\right|\right) \\
M_{c}^{-}=\frac{1}{2}\left(m^{+}+m^{-}-\left|m^{+}+m^{-}\right|\right) \\
m^{+}= \begin{cases}0 & \text { if } M_{L} \leq-1 ; \\
\frac{1}{4}\left(M_{L}+1\right)^{2} & \text { if }-1<M_{L}<1 ; \\
M_{L} & \text { if } M_{L} \geq 1 ;\end{cases} \\
m^{-}= \begin{cases}M_{R} & \text { if } M_{R} \leq-1 ; \\
-\frac{1}{4}\left(M_{R}-1\right)^{2} & \text { if }-1<M_{R}<1 ; \\
0 & \text { if } M_{R} \geq 1 ;\end{cases} \\
M_{p}^{+}= \begin{cases}0 & \text { if } M_{L} \leq-1 ; \\
\frac{1}{4}\left(M_{L}+1\right)^{2}\left(2-M_{L}\right) & \text { if }-1<M_{L}<1 ; \\
1 & \text { if } M_{L} \geq 1 ;\end{cases}
\end{gathered}
$$

$$
M_{p}^{-}= \begin{cases}1 & \text { if } M_{R} \leq-1 \\ \frac{1}{4}\left(M_{R}-1\right)^{2}\left(2+M_{R}\right) & \text { if }-1<M_{R}<1 \\ 0 & \text { if } M_{R} \geq 1 ;\end{cases}
$$

While the AUSM scheme has the much desired simplicity of flux-vector splitting schemes, its accuracy has been shown to rival and even exceed that of the best flux-difference splitting schemes. In Ref. 11, the AUSM scheme was used to compute high Mach number flows with great accuracy. The flows computed included a supersonic flow over a blunt body with a detached shock. In Ref. 14, the AUSM scheme was used in an early version of the TRAF3D.MB code to compute a three-dimensional, low Mach number flow in a backward facing step. Again the results obtained proved highly accurate. Together, the computations presented in Refs. 11 and 14 demonstrate the versatility of the AUSM scheme and its suitability for use in computations of complicated internal and external flows at both low and high Mach numbers.

\section{Time Stepping Scheme and}

\section{Convergence Acceleration Techniques}

Solutions to the discretized governing equations are obtained in TRAF3D.MB by marching to steady state with a four-stage Runge-Kutta time integration scheme. ${ }^{9,15}$ Convergence to steady state is accelerated by using (a) local time stepping, (b) implicit residual smoothing, ${ }^{9,15}$ and (c) multigrid scheme. ${ }^{9,15-18}$ The local time step sizes are computed considering both inviscid and viscous contributions. The multigrid scheme is implemented using full approximation storage (FAS). The full multigrid procedure (FMG) is also implemented to generate good initial conditions for the finest grids. In the current implementation of the multigrid scheme in TRAF3D.MB, full weighting is used to restrict the fine grid solution and residuals to the coarser grids, and tri-linear interpolation is used in the prolongation of coarse-grid corrections to the finer grids.

The multigrid convergence acceleration technique is very well suited for problems that require high resolution of the flow field. The reason for this advantage is that multigrid methods yield convergence rates that are essentially independent of the number of grid points. This means that convergence rates on the finest grids in a multigrid scheme are the same as those on the coarsest grids. This results in a reduction in CPU time by a factor of up to 10 compared to computations on the finest grid alone.

The convergence acceleration techniques employed in TRAF3D and TRAF3D.MB are described in Ref. 9. For further information on the multigrid techniques, see Refs. 15-18. 


\section{Multiblock Scheme}

\section{Multiblock Grid Systems}

The TRAF3D.MB code is designed to use multiblock grids like the one shown in Fig. 1. The grid system in Fig. 1 illustrates many of the features of high-quality multiblock grids. The geometry in Fig. 1 is a cylinder confined between two walls. The bold lines in the grid system indicate an interface between blocks. The topology of the grid system is an O-grid around the cylinder that is fitted within an $\mathrm{H}$-grid structure for the channel. Note the smoothness and orthogonality almost everywhere in the grid. Note also that in the grid system in Fig. 1, there are four special points, singularities, where more than four grid lines intersect. The occurrence of such singularities is rather typical in multiblock grid systems for complex geometries. However, they are generally few and located away from the boundaries of the domain where high gradients may be expected in the solutions. The singularities pose no special difficulties for most flow solvers. In particular, no special treatment is needed for the convection terms in most cell-centered schemes. On the other hand, in order to ensure conservation in the overall scheme, care must be exercised when evaluating diffusion terms for cells (or cell-faces) immediately adjacent to the singular point.

\section{Implementation}

The multiblock capability implemented in TRAF3D.MB is completely general in the sense that no limitation is on the number of blocks or how they can be connected. For storage, a single one-dimensional array is used for all the individual blocks, with pointers to the first element in each block. The code is configured to automatically order the blocks into memory. If enough memory is available, all blocks are stored in memory at all times. Otherwise, the code automatically separates the blocks into groups or clusters that fit into memory, one at a time. Block clusters that are not in menory are saved in a file (SSD or "scratch"). When needed, a total of three files are used for each level of the multigrid scheme, regardless of the number of blocks in the grid. Two files are used to store the numerical solution while one file is used to store the grid system and metric coefficients.

In TRAF3D.MB, ghost cells are used to accomplish the necessary communication between blocks in the multiblock grid system. Transfer of data between blocks involves loading into the appropriate ghost cells of each block the data from its neighboring blocks that the block needs access to. The data transfer is implemented such that if two neighboring blocks reside in memory at the same time (i.e., belong to the same cluster of blocks), then the data exported by one block is written directly into the appropriate ghost cells of the other block. On the other hand, if the two blocks are not in memory at the same time (not in the same block cluster), then the data exported by one block is written into a special array from where the second block can access the data when it is brought into memory.

The amount of data that needs to be transferred between blocks depends on the order of accuracy of the numerical scheme and the type of difference formulas being used. In TRAF3D.MB, second order accurate upwind differencing is used for most cases. Consequently, two layers of ghost cells are used around each block in the multiblock grid system, effectively creating an overlap of four cells between neighboring blocks. This overlap supports up to third order accurate upwind-biased difference formulas for all cells in the grid system without reduction in order of accuracy at block interfaces.

\section{Multiblock/Multigrid Scheme}

There are a number of possible ways to implement a multigrid scheme in the multiblock Runge-Kutta time stepping framework. The possible choices differ in when communication between blocks takes place; i.e., when transfer of data is done to update values in ghost cells at block interfaces. Ideally, communication between blocks should take place at all levels in the multigrid sequence and at each stage in the Runge-Kutta scheme. Furthermore, communication should take place in the implicit residual smoothing operation to ensure maximum benefit of that operation. While being optimal for convergence, this ideal approach involves a large overhead due to transfer of data between blocks and may not be optimal with respect to overall CPU time requirements.

To reduce the overhead due to transfer of data between blocks, a compromise strategy is implemented in TRAF3D.MB. The strategy is to let blocks communicate on each level in the multigrid scheme but only before the first stage in the Runge-Kutta scheme, and to apply the residual smoothing independently within each block. This reduces significantly the overhead due to communication between blocks without significantly impacting the convergence rate.

\section{Results}

To test and demonstrate the usefulness of the TRAF3D.MB code, solutions to three problems were 
computed. These problems are (a) flow in a linear cascade composed of NACA0012 airfoils. (b) flow around a cylindrical pin extending between two parallel plates, and (c) flow of air through a coolant passage inside a turbine blade of a radial-inflow turbine. At this point, detailed comparison of the computed results to experimental data have not yet been done. In Ref. 11, however, results obtained with an earlier version of TRAF3D.MB for a three-dimensional flow over a backward-facing step were compared to experimental data. That the numerical results agreed very well with the experimental data is an indication of the accuracy of the present scheme.

\section{Flow in a Linear Cascade}

The first case to be discussed is a flow in a linear cascade composed of NACA0012 airfoils. The flow into the cascade was at zero angle of attack and Mach number of 0.5. The Reynolds number based on the speed of the incoming flow and cord length was $10^{6}$. The objective of computing this flow was to compare the convergence characteristics obtained using a multiblock grid to that obtained using a single-block grid. For this purpose, the multiblock grid was created by dividing the single-block grid up into three parts. Figure 2 shows the grid system for the cascade - the bold lines in the grid indicate where the single block was divided up to create the multiblock grid. The grid consisted of a $153 \times 41$ two-dimensional (2-D) grid stacked up in 33 layers from end wall to symmetry plane to create a three-dimensional (3-D) grid. Grid points were clustered near the airfoil surface and near the end wall. No-slip, adiabatic wall boundary conditions were applied at the airfoil surface and at the end wall.

To compare the convergence characteristics of the multiblock scheme to that obtained by using a single block, the same full multigrid procedure was applied to both; i.e., 100 iterations were performed on each of the three grid levels, starting out with a uniform flow on the coarsest grid. A three-level "sawtooth" multigrid cycle was used on the finest grid with two iterations on the intermediate grid level and three iterations on the coarsest grid level. The convergence history of the computations is plotted in Fig. 3, whereas contours of axial velocity components are shown in Fig. 4. As Fig. 3 shows, the convergence histories for the single-block and multiblock schemes are virtually identical. Note, the spikes observed in the grapl occurred at iterations 101 and 201, when the full multigrid method proceeded from a coarser grid to a finer one. While the convergence histories were nearly identical, the CPU and memory requirements for the two cases were not the same. The multiblock computations consumed about $10 \%$ more CPU time than the single block computations but used about $25 \%$ less memory, even while storing all blocks and grid levels in memory at all times. The total CPU time for the single-block run was about $30 \mathrm{~min}$. on a CRAY YMP (single processor), or 8.4 . $10^{-5} \mathrm{CPU}$ sec. pr. grid point pr. iteration for a threelevel multigrid cycle.

\section{Pin in a High Aspect Ratio Channel}

The second solution to be presented is for a flow around a cylindrical pin extending between the two main walls in a high aspect ratio channel. The height of the channel (length of the pin) was taken to be equal to the diameter of the pin, and the width of the channel was 16 times the diameter of the pin. Figure 1 shows the grid system in one plane of the channel. The 3-D grid system was generated by stacking the 2-D grids. A total of 33 planes were used from one wall to the symmetry plane of the channel. The multiblock grid system consists of a total of 12 blocks, with $33 \times 33 \times 33$ grid points in the four blocks in the corners of the geometry (See Figure 1) and $17 \times 33 \times 33$ points in the remaining blocks. The coordinate system is oriented such that the $\mathrm{x}$-coordinate is parallel to the main flow direction, the $y$-coordinate is from side wall to side wall, and the $\mathrm{z}$-coordinate is from the floor to the symmetry plane. The flow conditions were chosen to approximate conditions within a coolant passage of a turbine blade, with Mach number of 0.2 at the entrance of the channel and Reynolds number of 3,000 (based on diameter of the pin and speed of the incoming flow). Turbulent flow was assumed with the Baldwin-Lomax eddy viscosity model applied at the main walls of the channel.

Figures 5-8 show the solution for the flow around the pin. Figures 5 and 6 show the contours of $u$-velocity, $v$-velocity and Mach number in the symmetry plane between the two main walls of the channel and near the wall, respectively. As Fig. 5 shows, the flow separates behind the pin. The separation point is found to be at $87^{\circ}$ from the forward stagnation point. Figure 6 shows that in front and back of the pin the fluid is flowing away from the pin-opposite to the direction of the flow in the symmetry plane (see Fig. 5). This flow is the result of the "down-wash" of the high momentum fluid in the center plane of the channel after it has impinged on the pin in front and back. Overall, Figs. 5 and 6 show the excellent symmetry that is maintained in the solution. This symmetry is an indication of careful implementation of the numerical method of solution since the flow that is being simulated has a natural tendency to be unsteady and asymmetric. Indeed, if slight 
asymmetry is introduced into the grid or boundary conditions asymmetry arises quickly in the solution leading to strong oscillations in the location of the separation points on the pin and, subsequently, to vortex shedding. No attempt was made to capture this unsteady phenomenon in the present study since highlly refined grids are needed to accurately capture the shedding of vortices.

Figures 7 and 8 show the flow in the corner between the pin and the channel wall. Figure 7 shows the solution in front of the pin and Fig. 8 shows the solution at $90^{\circ}$ below the forward stagnation point. The dimensions that are shown on the axes in the figures have been normalized by the half-width of the channel. In the figures, the vertical axes correspond to the surface of the pin (z-axis), whereas the horizontal axes correspond to the channel floor ( $x$-axis in Fig. 7, $y$-axis in Fig. 8). Together, the figures show the horse-shoe vortex that forms in the corner between the pin and the wall. Figure 7 shows the vortex that is created in front of the pin in the corner between the channel and the pin due to the down-wash from the mid plane of the channel towards the channel floor. Figure 8 shows that $90^{\circ}$ below the forward stagnation point, the strength and size of the recirculation has increased.

\section{Flow in radial turbine coolant passage}

The last solution to be presented is for a flow of air through a coolant passage inside a turbine blade of a radial-inflow turbine. The geometry of the coolant passage is shown in Fig. 9. As the figure indicates, the geometry of the passage is complicated, with important small-scale features such as the pins that extend from wall to wall in the passage. The grid system used in the computations of the flow in the passage is shown in Fig. 10. The generation of this grid system was discussed in Ref. 19. Slight modification has heen made in the grid system from that presented in Ref. 19. to better model the inflow geometry. The bold lines in Fig. 10a indicate a block boundary in the multiblock grid. A total of 6 blocks were used with a total of 97,440 grid points. The flow conditions specified correspond to inflow stagnation pressure and temperature of $1.6 \mathrm{bar}$ and $300 \mathrm{~K}$ respectively, and with the back pressure at the outflow boundary specified at 0.65 times the inflow stagnation pressure.

Since the smoothness and resolution of the grid system is substantially inadequate for accurate computations, only preliminary computations were done using first order upwind differencing for the convection terms of the governing equations. However, a grid refinement study for the first block of the coolant passage grid system confirms that the qualitative features of the flow in the present calcuation are correct.

Figures 11 and 12 show the computed flow field in the radial turbine coolant passage. Figure 11 shows the Mach number in the channel in a surface midway between the main blade surfaces. As the figure shows, the Mach number in the channel reaches about unity near the $180^{\circ}$ turn in the channel. In the latter half of the channel--i.e., after the sudden expansion where the first row of pins is located-the Mach number is considerably lower and typically around 0.1 .

Figures 12 shows the velocity vectors in the mid surface of the channel. The figure reveals a region of separated flow right after the $180^{\circ}$ turn. This is in sharp contrast to the results presented in Ref. 20 for the same geometry and the same grid, where no separation was predicted. The essential difference between the numerical methods employed in the current study and that employed in Ref. 20 lies in the scheme used for the inviscid fluxes. In Ref. 20 , some flux vector splitting (FVS) schenes were tested including van Leer's FVS scheme. In that study, second-order upwinding was used. The fact that the current AUSM scheme predicts separation, even with first-order upwinding, is an indication of the superiority of the scheme over the older FVS schemes.

Work is currently in progress on generating new solutions on finer grid systems for the coolant passage shown in Fig. 9.

\section{Conclusions}

Numerical simulations of viscous flows in complex geometries pose a triple challenge to Computational Fluid Dynamics. First, grid generation tools must be capable of generating high quality grid systems with minimal human intervention. Second, the discretizations used for the governing equations must be capable of yielding accurate solutions to flows with complex physics, such as turbulence, flow separation, large variations in Mach number, shocks, and high temperature gradients. Finally, the the flow solvers must be computationally efficient since a large number of grid points is typically needed to accurately resolve small features in the geometries and the flow fields.

In this study; a computer program has been developed that is capable of accurate simulations of viscous flows in complex geometries. To handle the complex geometries, the code uses multiblock grid systems that can, with the aid of specialized software, be generated for geometries of arbitrary complexity. The code uses 
the AUSM scheme to discretize the convection terms of the Navier-Stokes Equation. This scheme has been shown to be highly accurate in a varyity of flow conditions. Finally, to achieve the needed efficiency the code uses a multigrid scheme along with other techniques to accelerate convergence of computed solutions to steady state.

The development of the TRAF3D.MB code is continuing. Planned and ongoing work includes implementation of a two-equation turbulence model, implementation of the code on parallel computer architectures, and further validation of the code.

\section{Acknowledgements}

The multiblock grid system in Fig. 1 was generated by Program Development Corporation of White Plains, New York, and the grid in Fig. 2 was generated by Dr. David L. Rigby, Sverdrup Technology, Inc. Cleveland, Ohio. The authors are grateful to the individuals involved for their contribution.

\section{References}

1 Eiseman, P. R., and Erlebacher, G., "Grid Generation for the Solution of Partial Differential Equations", NASA CR-178365, 1987.

2 Aftomis, M. J., "Viscous Flow Simulation Using an Upwind Method for Hexahedral Based Adaptive Meshes," AIAA 93-0772, January 1993.

3 Mathur, S. R., Madavan, N. K., and Rajagopalan, R. G., "A Hybrid Structured-Unstructured Grid Method for Unsteady Turbomachinery Flow Computations," AIAA 93-0387, January 1993.

4 Mavriplis, D., "Turbulent Flow Calculations Using Unstructured and Adaptive Meshes," ICASA Report No. 90-61.

5 Allwright, S. E., "Techniques in Multiblock Domain Decomposition and Surface Grid Generation," in $\mathrm{Nu}$ merical Grid Generation for Computational Fluid Mechanics '88; Sengupta, S., Häuser, J., Eiseman, P. R., and Thompson, J. F., (ed), pp. 559-568, Pineridge Press, 1988.

6 Amdahl, D. J., "Interactive Multi-Block Grid Generation," in Numerical Grid Generation for Computational Fluid Mechanics '88; Sengupta, S., Häuser, J., Eiseman, P. R., and Thompson, J. F., (ed), pp. 579-578, Pineridge Press, 1988.
7 Stewart, M., "A General Decomposition Algorithm Applied to Multi-Element Airfoil Grids," AIAA 90-1606, 1990.

8 "GRIDPRO,TM /AZ3000, Users Guide and Reference Manual", Program Development Corporation, White Plains, NY, 1993.

9 Arnone, A., Liou, M.-S., and Povinelli, L. A., "Multigrid Calculation of Three-Dimensional Viscous Cascade Flows," AIAA-91-3238, Sept. 1991.

10 Arnone, A., Liou, M.-S., and Povinelli, L. A., "NavierStokes Solution of Transonic Cascade Flows Using NonPeriodic C-Type Grids," Journal of Propulsion and Power, Vol. 8, 1992, pp. 410-417.

11 Liou, M.-S., and Steffen, C. J., "A New Flux Splitting Scheme," NASA TM 104404, May 1991. Also, J. of Comp. Physics, Vol. 107, pp.23-39, 1993.

12 Schlichting, H., "Boundary-Layer Theory," McGrawHill, New York, 1979.

13 Baldwin, B. S., and Lomax, H., "Thin Layer Approximation and Algebraic Model for Separated Turbulent Flows," AIAA paper 78-257, 1978.

14 Steinthorsson, E., Liou, M.-S., Povinelli, L. A. and Arnone, A., "Numerical Simulations of Three-Dimensional Laminar Flow Over A Backward Facing Step; Flow Near Side Walls," FED Vol 149, pp. 19-26, ASME 1993.

15 Jameson, A., "Transonic Flow Calculations," MAE Report 1651, MAE Department, Princeton University, July 1983.

16 Brandt, A., 'Multi-level Adaptive Solutions To Boundary Value Problems," Mathem. of Computation, Vol. 31, pp. 333-390, 1977.

17 Hackbusch W., and Trottenberg, U., "Multigrid Methods," Springer-Verlag, Berlin, 1982.

18 Briggs, W. L., "A Multigrid Tutorial," SIAM, Philadelphia, Pennsylvania 1987.

19 Steinthorsson, E., Shih, T. I-P., and Roelke, R. J., "Algebraic Grid Generation for Coolant Passages of Turbine Blades with Serpentine Channels and Pin Fins," AIAA 91-2366, June, 1991.

20 Steinthorsson, E., Shih, T. I-P., and Roelke, R. J., "Computation of the Three-Dimensional Flow and Heat Transfer Within a Coolant Passage of a Radial Turbine," AIAA-91-2238, June, 1991. 


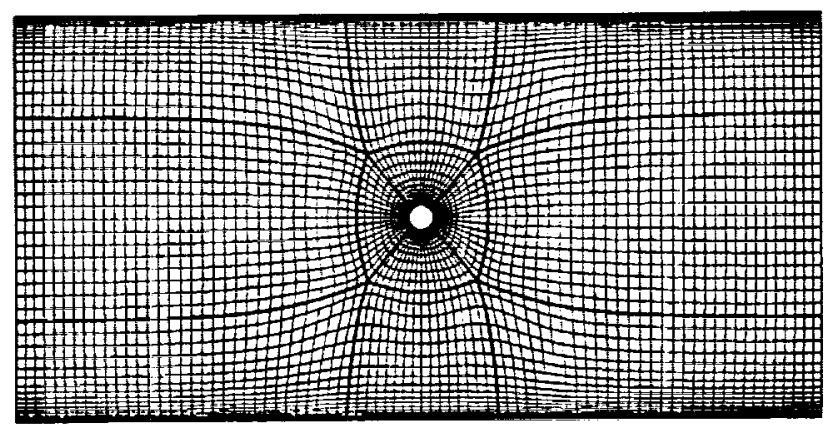

Figure 1. A multiblock grid system in 12 blocks: An O-grid topology within an H-grid topology

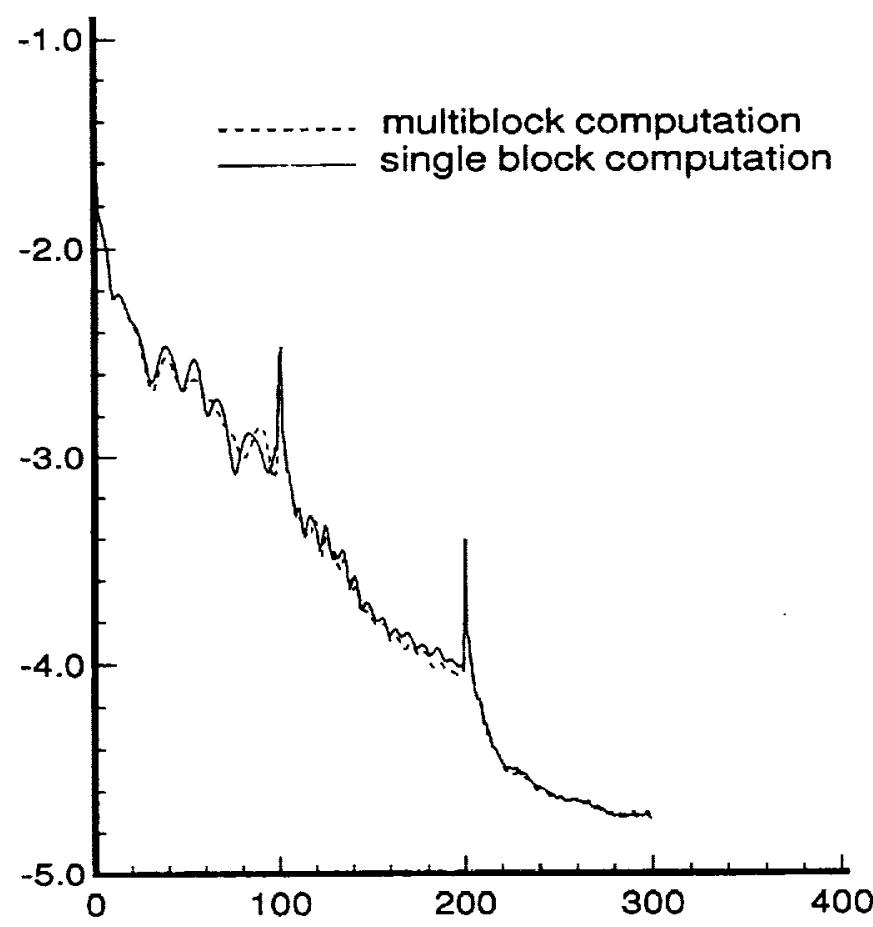

Figure 3. Convergence history for viscous flow in a linear cascade $\left(M=0.5, R e=10^{\circ}\right.$, zero angle of attack) - root-mean-square of density residual versus iterations

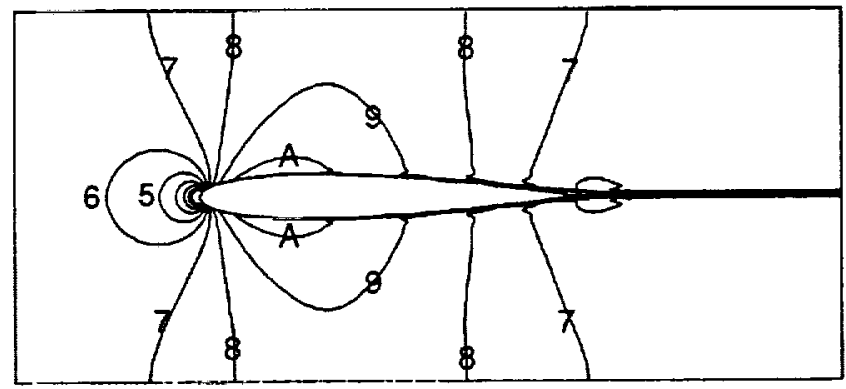

Figure 4. Flow in a linear cascade $(M=0.5, R e=$ $10^{6}$, zero angle of attack): Contours of normalized axial velocity, $u$, in plane of symmetry. $v_{1}=0.6, \Delta u=$ 0.075 .
Figure 2. A multiblock grid system in 3 blocks around a NACA0012 airfoil in a linear cascade: Bold lines indicate block boundaries-merging of block creates a single-block C-grid.

block 3: $49 \times 41$

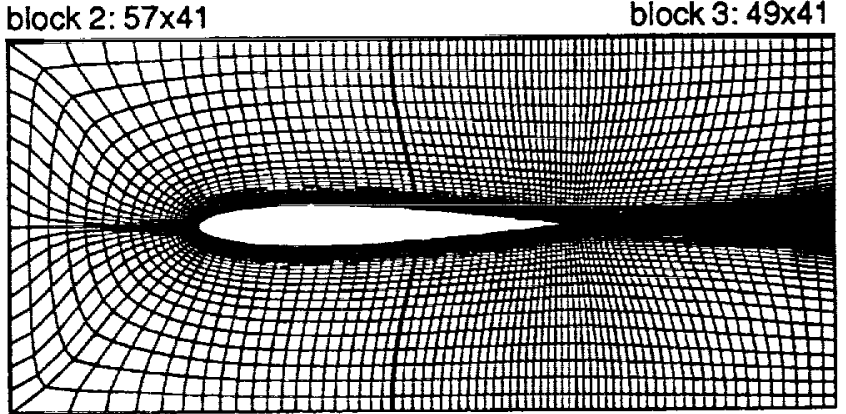

block 1: $49 \times 4$ 


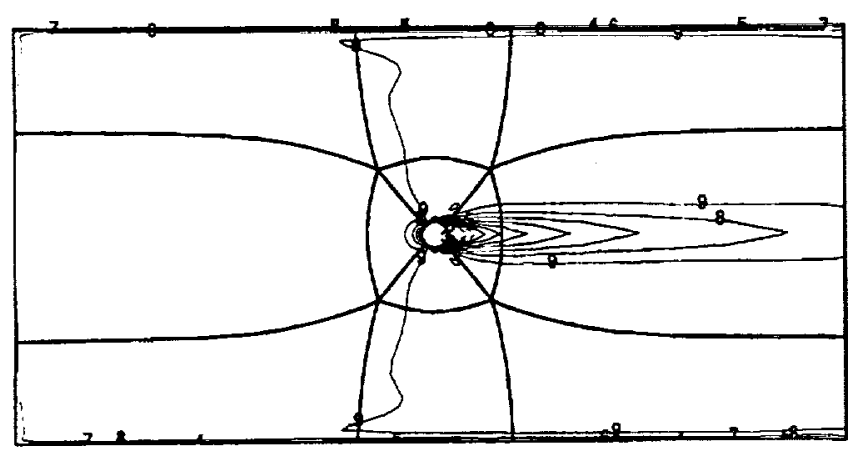

(a)

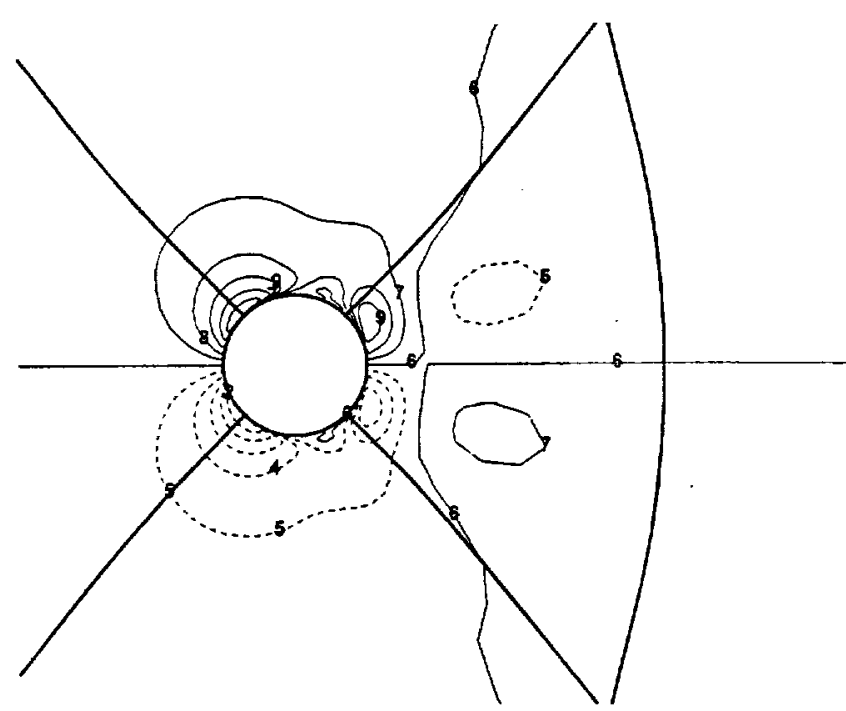

(c)

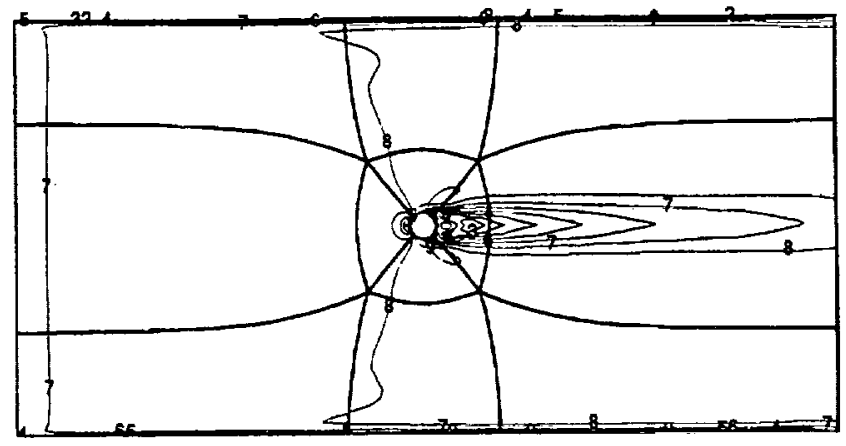

(b)

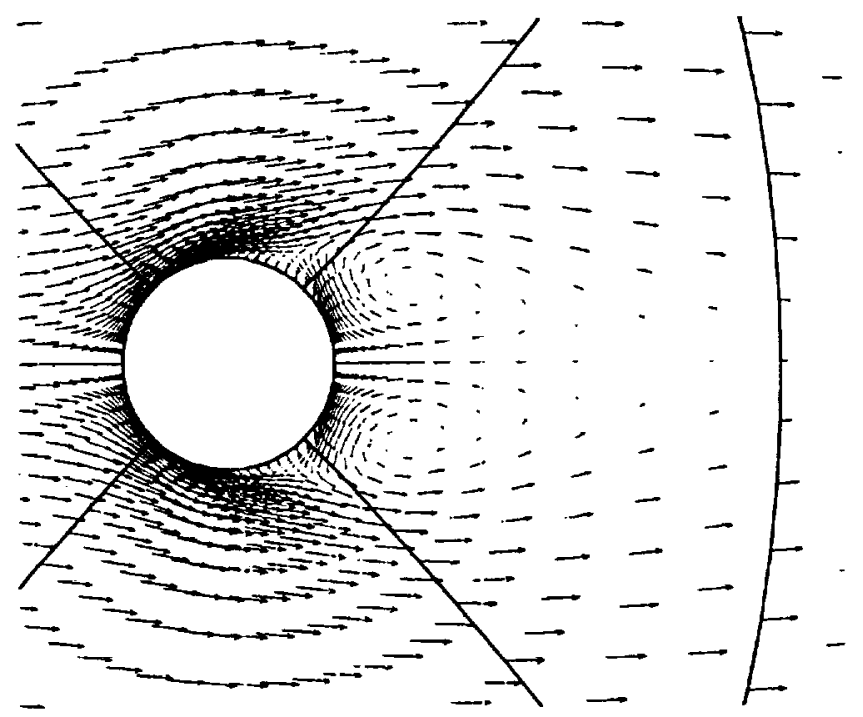

(d)

Figure 5. Pin in a high aspect-ratio channel: Flow field in symmetry plane

(a) Contours of normalized $x$-component of velocity $(u) ; u=-0.4, \Delta u=0.2$.

(b) Contours of Mach number, $M ; M_{1}=0, \Delta M=0.03$.

(c) Contours of normalized $y$-component of velocity $(v)$ near pin; $y=-0.7, \Delta v=0.14$.

(d) Velocity vectors near pin. 


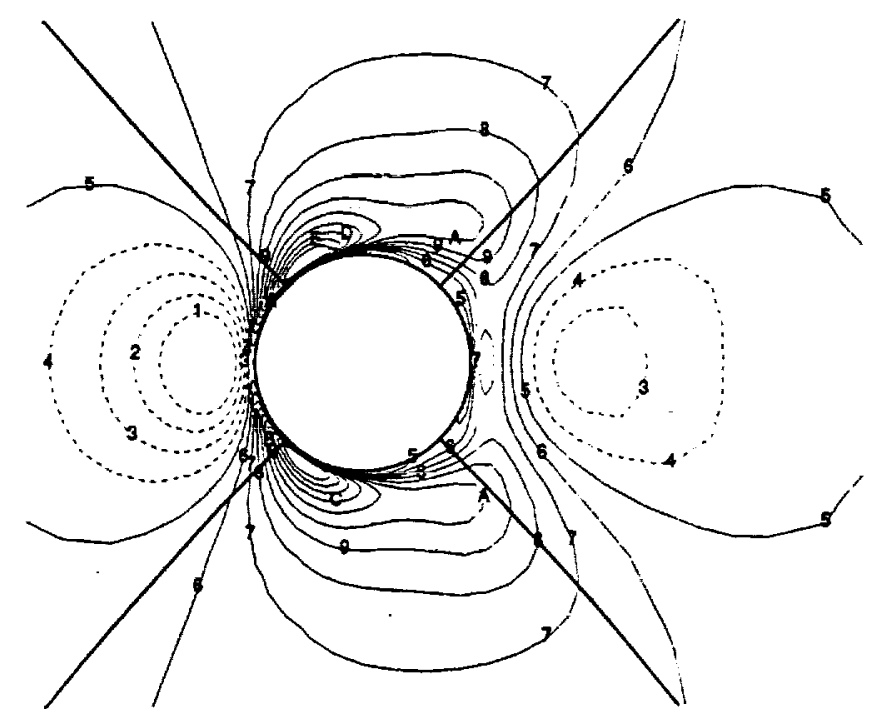

(a)

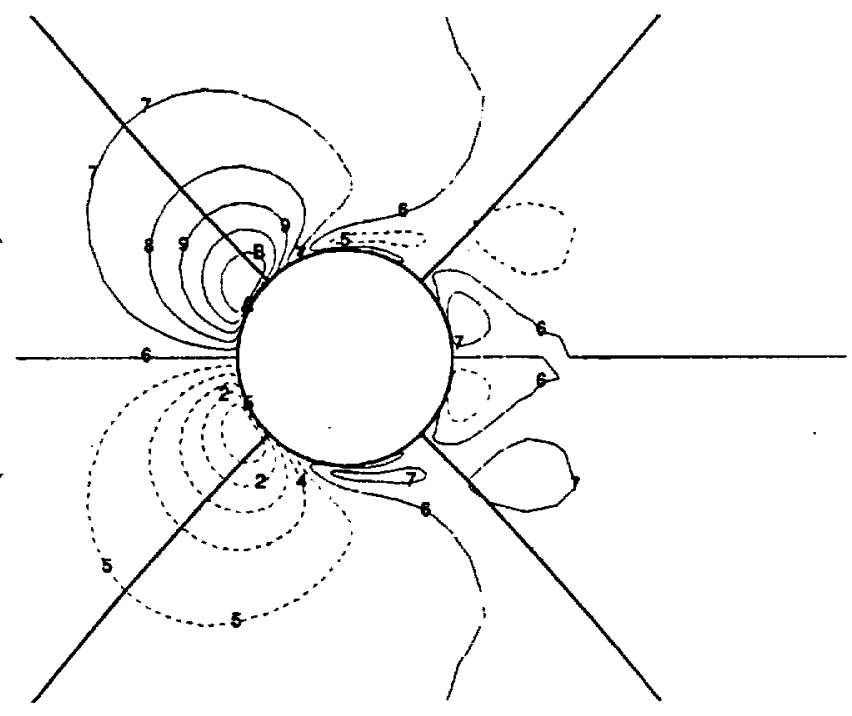

(b)

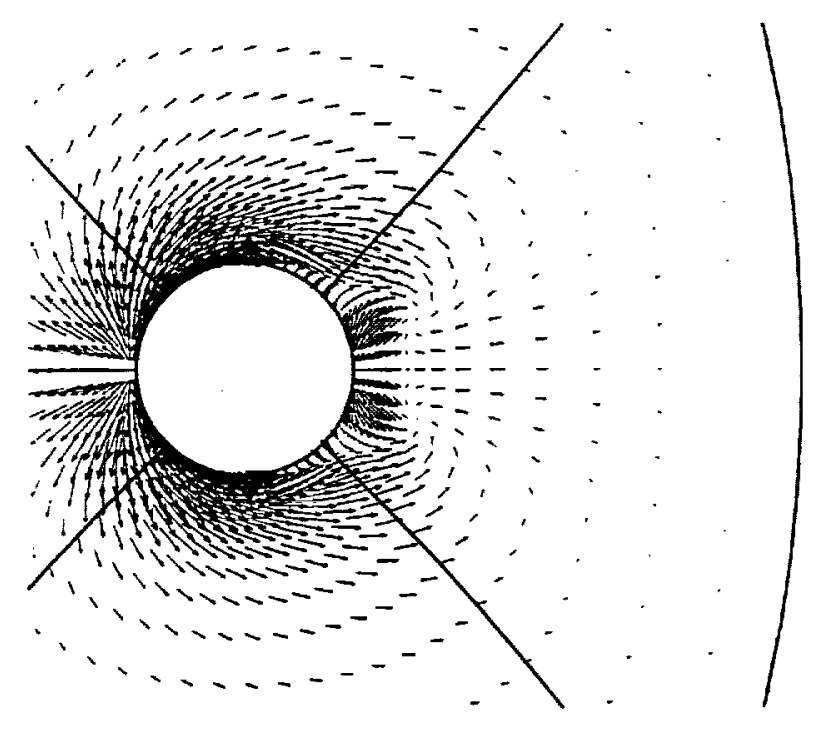

(c)

Figure 6. Pin in a high aspect-ratio channel: Flow field near pin adjacent to channel floor.

(a) Contours of normalized $x$-component of velocity $(u)$; $u=-0.008, \Delta u=0.002$.

(b) Contours of normalized $y$-component of velocity $(v) ; y=-0.015, \Delta v=0.003$.

(c) Velocity vectors. 


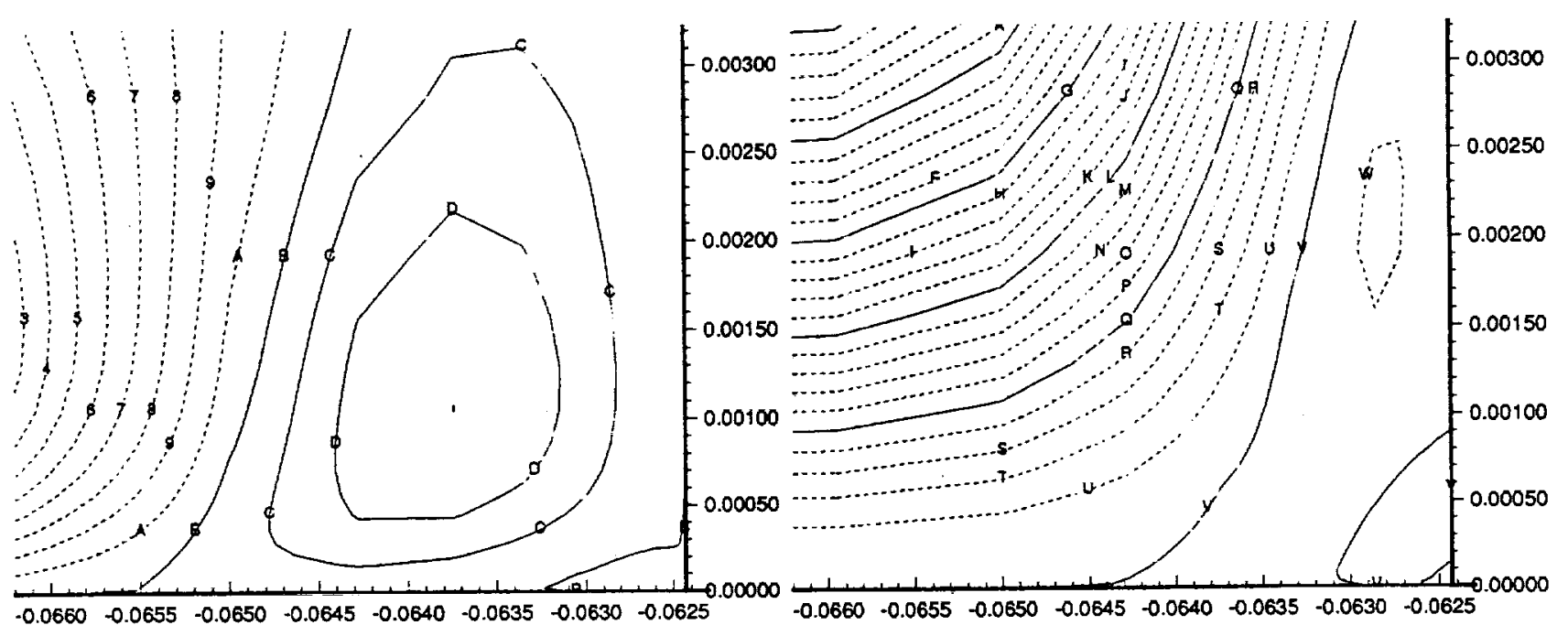

(a)

(b)

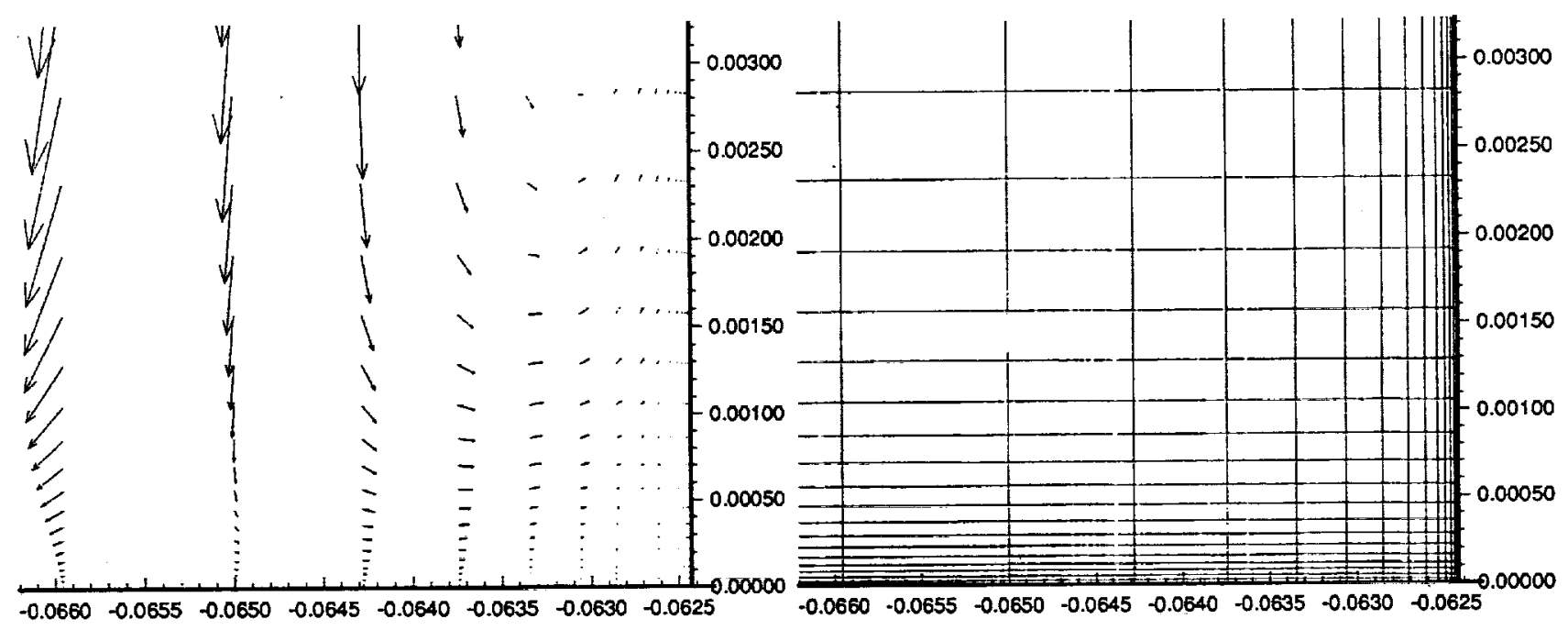

(c)

(d)

Figure 7. Pin in a high aspect-ratio channel: Flow field in corner between pin and channel floor in the symmetry plane front of forward stagnation point.

(a) Contours of normalized $x$-component of velocity $(u) ; u=-0.05, \Delta u=0.005$.

(b) Contours of normalized $z$-component of velocity $(w)$; $\imath$ - $=-0.18, \Delta w=0.006$.

(c) Velocity vectors. (d) Grid system. 


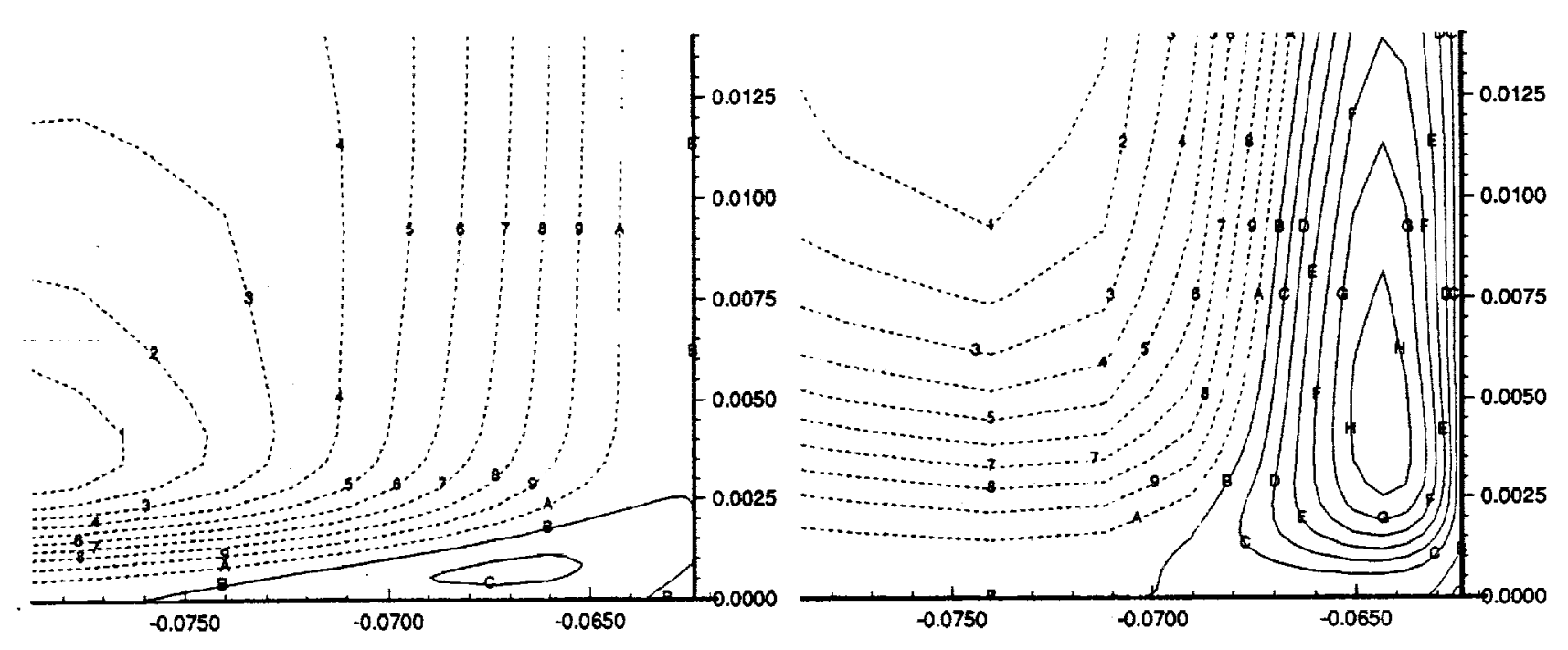

(a)

(b)

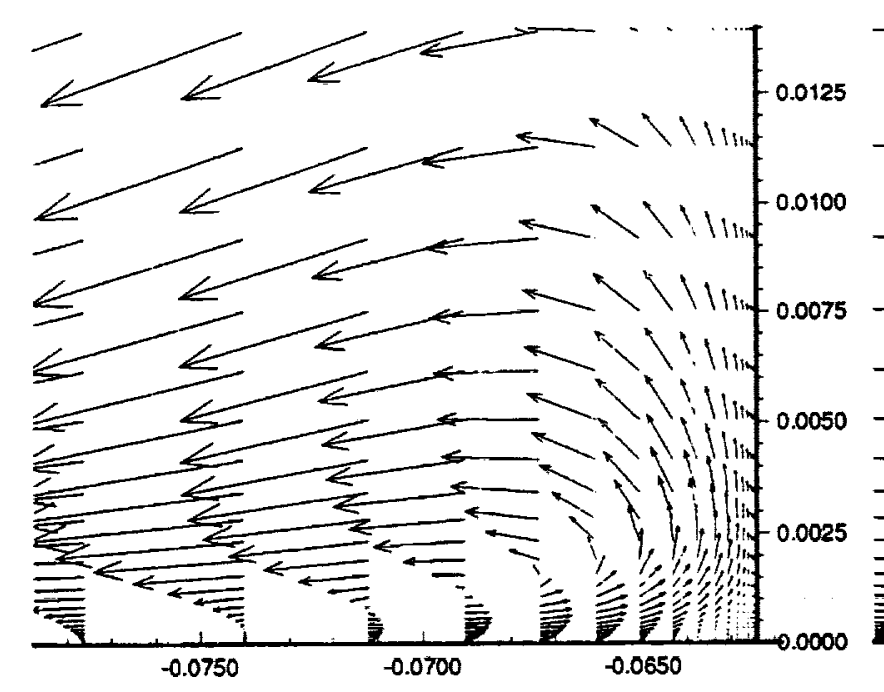

(c)

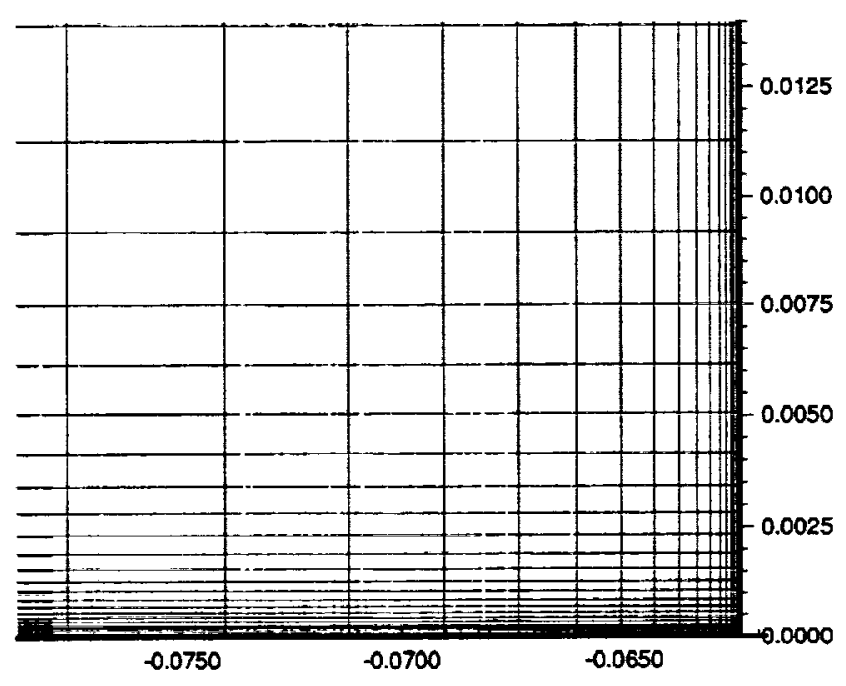

(d)

Figure 8. Pin in a high aspect-ratio channel: Flow field in corner between pin and channel floor in cross section $90 \mathrm{deg}$ below forward stagnation point.

(a) Contours of normalized $y$-component of velocity $(v) ; y=-0.4, \Delta v=0.04$.

(b) Contours of normalized $z$-component of velocity $(w)$; $\imath_{1}=-0.1, \Delta w=0.01$.

(c) Velocity vectors. (d) Grid system. 


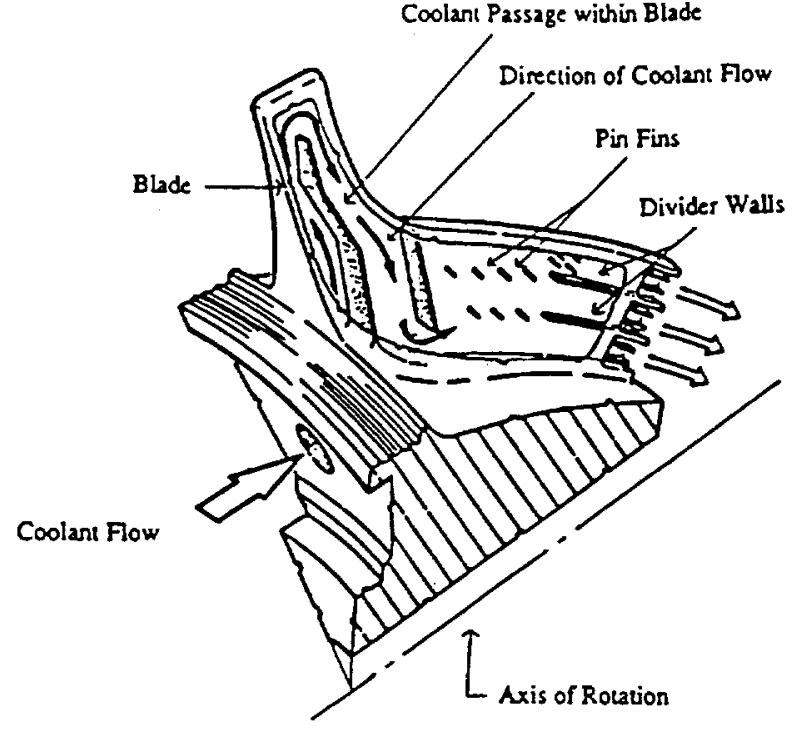

(a)

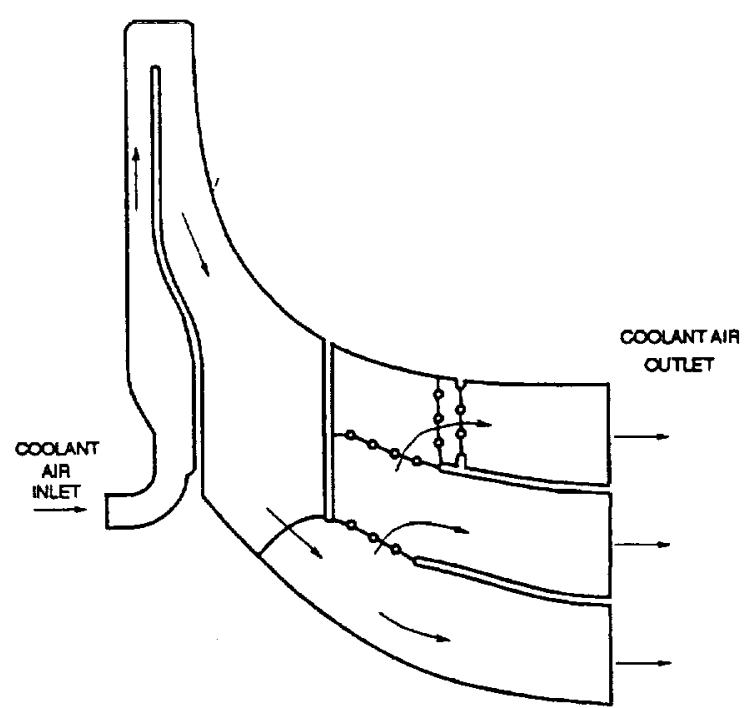

(b)

Figure 9. Coolant passage inside a blade of a radial turbine: (a) A cut-out view. (b) A schematic drawing.

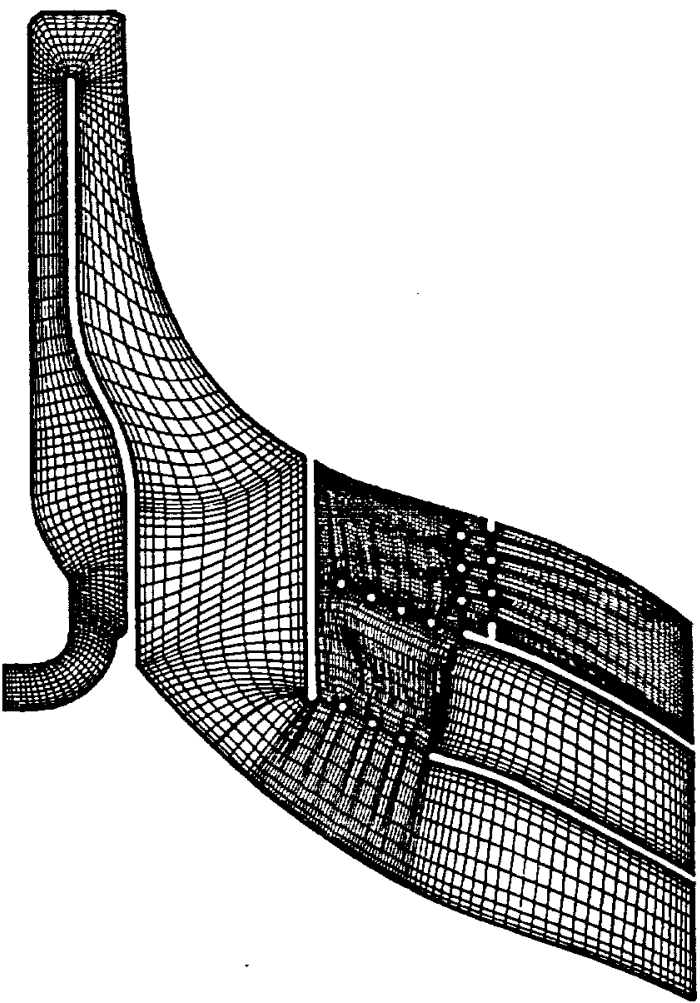

(a)

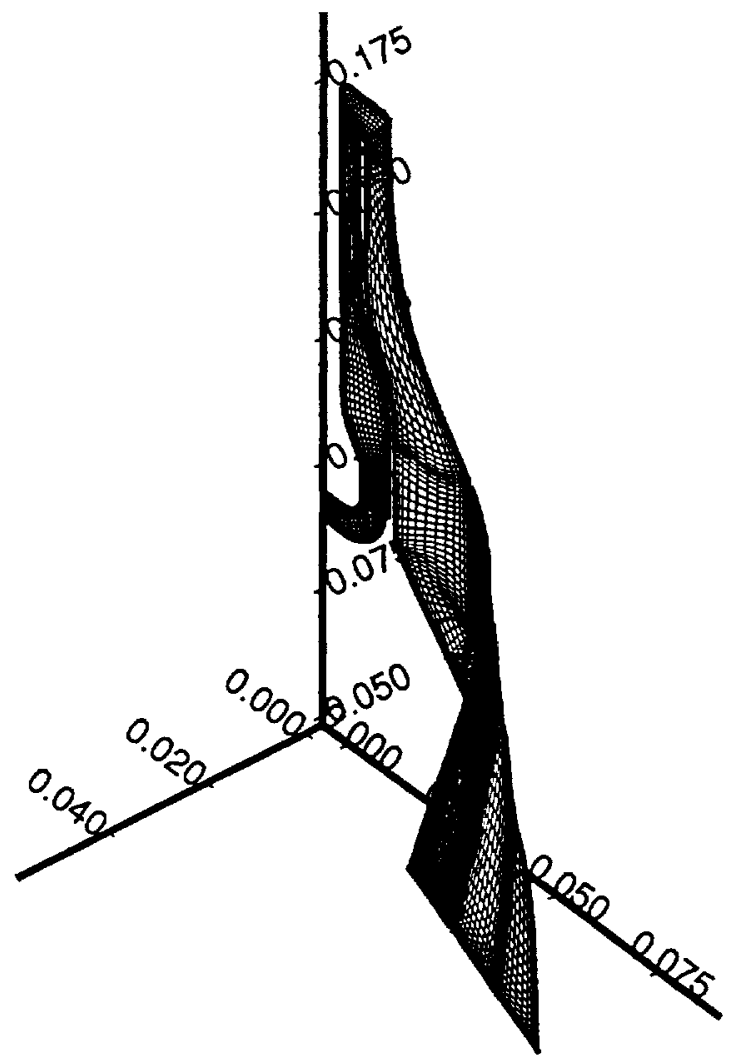

(b)

Figure 10. Grid system for radial turbine coolant passage-grid surface in the midplane of the passage: (a) A two-dimensional view. (b) A three-dimensional view. 


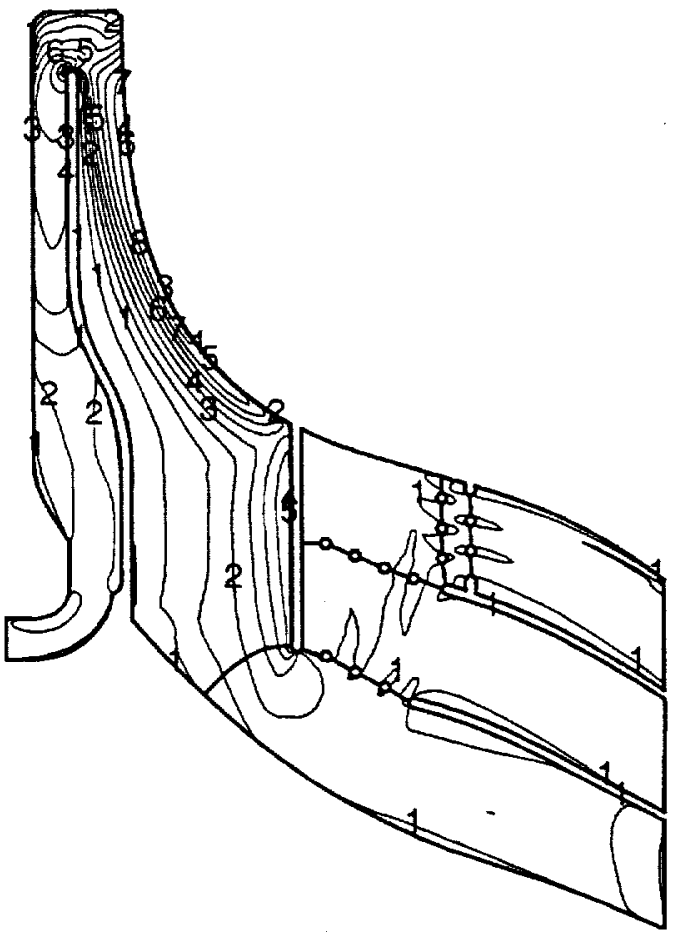

(a)

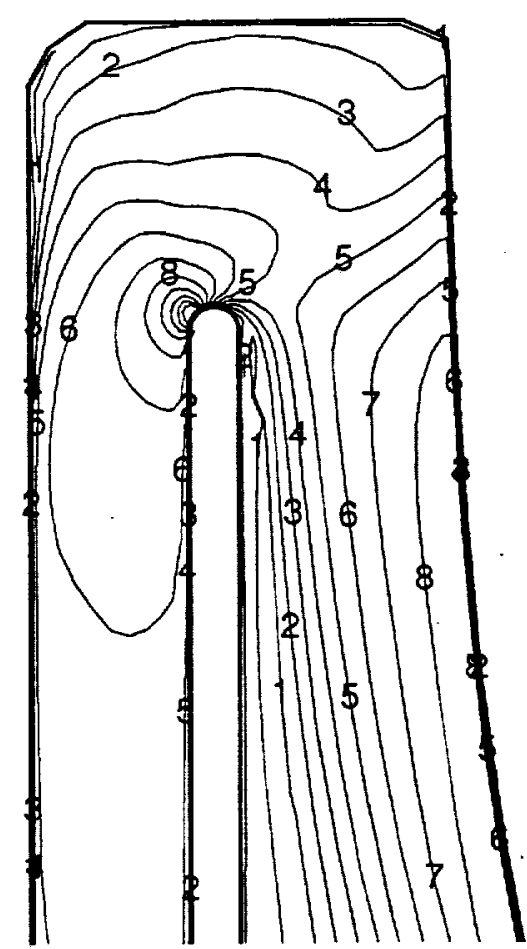

(b)

Figure 11. Mach number contours in the mid plane of the radial turbine coolant passage: $M_{1}=0.05, \Delta M=0.075$

(a) Overall view. (b) Close up view of $180^{\circ}$ turn. 


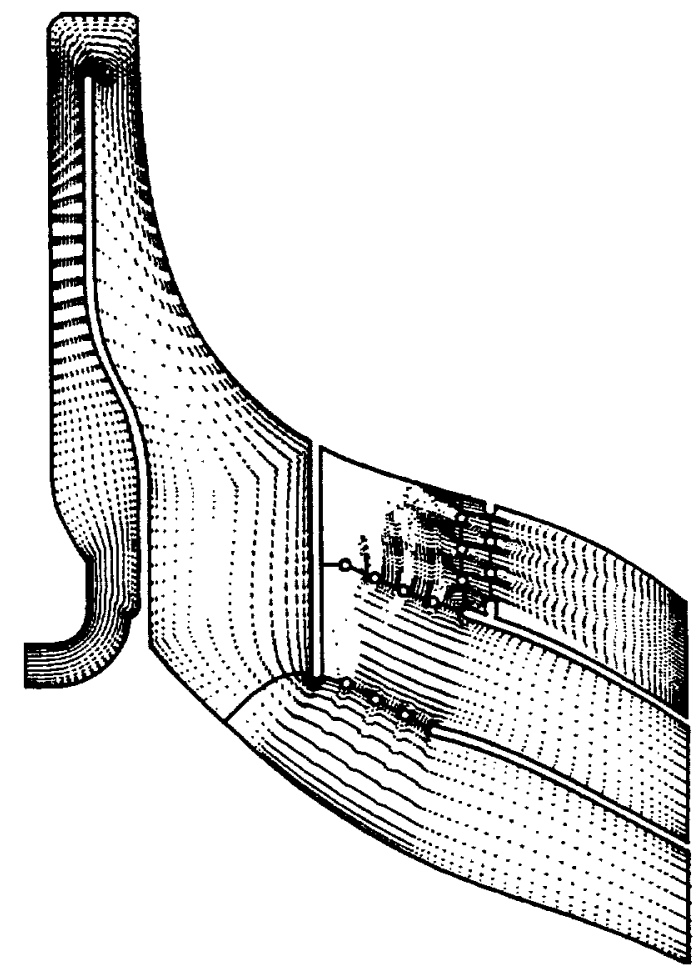

(a)

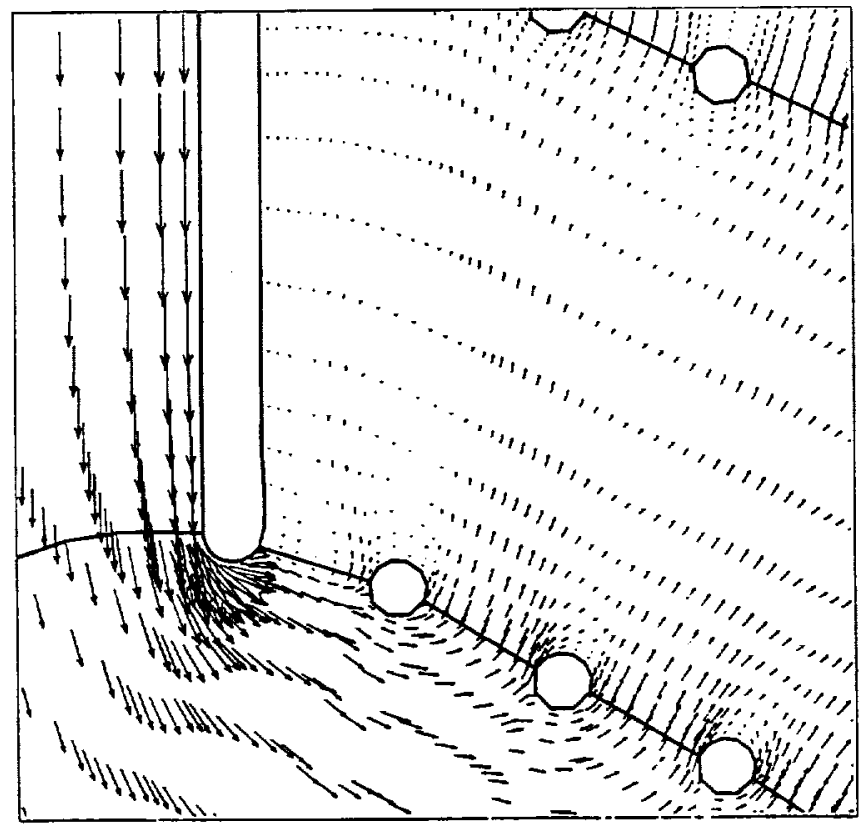

(c)

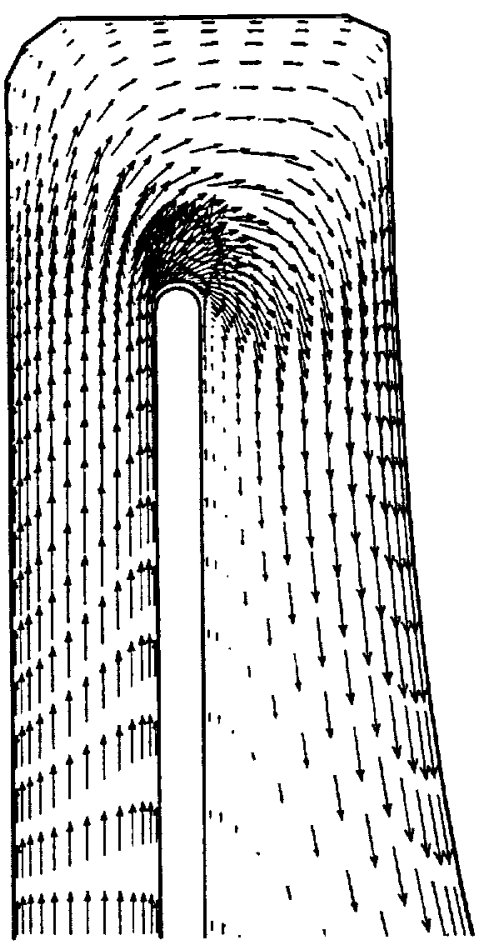

(b)

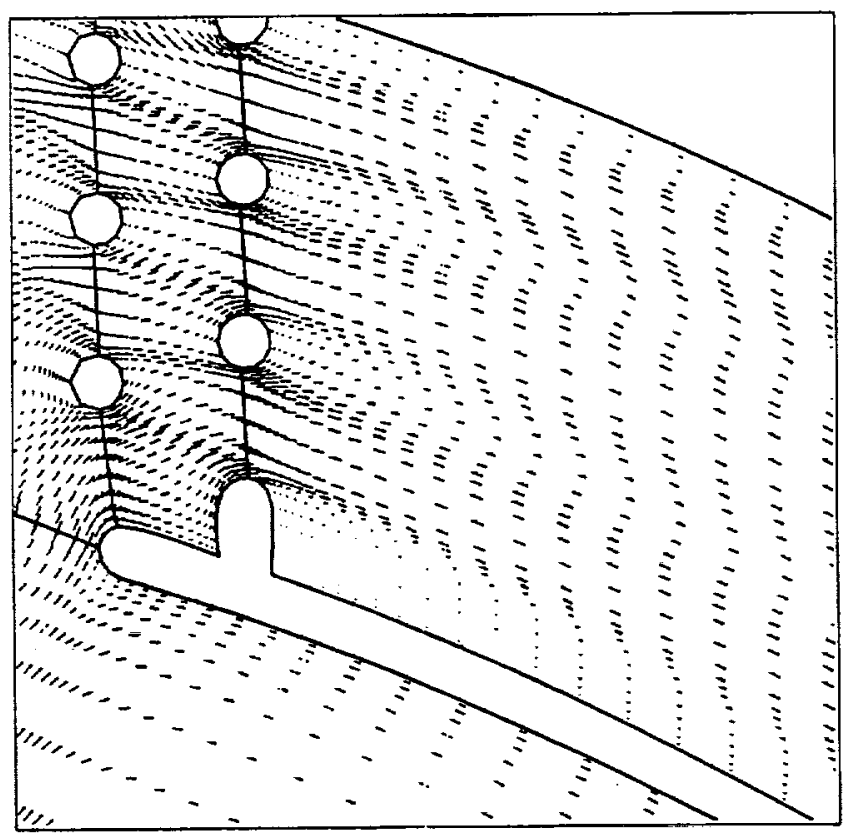

(d)

Figure 12. Velocity field in the mid plane of the radial turbine coolant passage:

(a) Overall view. (b) Close up view of $180^{\circ}$ turn. (c) Close up view of second turn and first array of pinfins.

(d) Closep view of last array of pinfins 
Public reporting burden for this collection of intormation is estimated to average 1 hour per response, including the time lor reviewing instructions, searching extsting data sources, gathering and maimaining the data needed, and completing and reviewing the collection of information. Send comments regarding this burden estimale or any other aspect of this collection of intormation, including suggestions for reducing this burden, to Washington Headquanters Services, Directorate for Intormation Operations and Reports. 1215 Jefferson Davis Highway, Suite 1204. Arlington, VA 22202-4302, and to the Ottice of Management and Budget, Paperwork Reduction Project (0704-0188), Washington, DC 20503.

1. AGENCY USE ONLY (Leave blank)

\section{REPOAT DATE}

June 1993

\section{REPORT TYPE AND DATES COVERED}

Technical Memorandum

\section{TITLE AND SUBTITLE}

Development of an Explicit Multiblock/Multigrid Flow Solver for Viscous Flows in Complex Geometries

\section{AUTHOR(S)}

E. Steinthorsson, M.S. Liou, and L.A. Povinelli

\section{PERFORMING ORGANIZATION NAME(S) AND ADDRESS(ES)}

National Aeronautics and Space Administration

Lewis Research Center

Cleveland, Ohio 44135-3191

9. SPONSORING/MONITORING AGENCY NAME(S) AND ADDRESS(ES)

National Aeronautics and Space Administration

Washington, D.C. 20546-0001

\section{FUNDING NUMBERS}

WU-505-62-27

8. PERFormang ORGANIzATION REPORT NUMBER

E-8140

10. SPONSORINGMONITORING AGENCY REPORT NUMBER

NASA TM-106356

ICOMP-93-34

AIAA-93-2380

11. SUPPLEMENTARY NOTES

Prepared for the 29th Joint Propulsion Conference and Exhibit cosponsored by AIAA, SAE, ASME, and ASEE, Monterey, Califomia, June 28-30,

1993. M.S. Liou and L.A. Povinelli, NASA Lewis Research Center and E. Steinthorsson, Institute for Computational Mechanics in Propulsion,

NASA Lewis Research Center, (work funded under NASA Cooperative Agreement NCC3-233). ICOMP Program Director, Louis A. Povinelli, (216) 433-5818.

12a. DISTRIBUTTONAVALABILTY STATEMENT

12b. DISTRIBUTION CODE

Unclassified -Unlimited

Subject Category 34

13. ABSTRACT (Maximum 200 words)

A new computer program is being developed for doing accurate simulations of compressible viscous flows in complex geometries. The code employes the full compressible Navier-Stokes equations. The eddy viscosity model of Baldwin and Lomax is used to model the effects of turbulence on the flow. A cell centered finite volume discretization is used for all terms in the governing equations. The Advection Upwind Splitting Method (AUSM) is used to compute the inviscid fluxes, while central differencing is used for the diffusive fluxes. A four-stage Runge-Kutta time integration scheme is used to march solutions to steady state, while convergence is enhanced by a multigrid scheme, local time-stepping and implicit residual smoothing. To enable simulations of flows in complex geometries, the code uses composite structured grid systems where all grid lines are continuous at block boundaries (multiblock grids). Example results are shown a flow in a linear cascade, a flow around a circular pin extending between the main walls in a high aspect-ratio channel, and a flow of air in a radial turbine coolant passage.

\section{SUBJECT TERMS}

Multiblock grid systems; Multigrid algorithm; Upwind differencing

17. SECURTYY CLASSIFICATION OF REPORT Unclassified
18. SECURTY CLASSIFICATION OF THIS PAGE Unclassified
19. SECURTY CLASSIFICATION OF ABSTRACT Unclassified 